\title{
Reticulocyte count and its parameters: comparison of automated analyzers, flow cytometry, and manual method
}

\author{
Varun Uppal $^{1} \cdot$ Shano Naseem ${ }^{1}$ (D) $\cdot$ Ishwar Bihana ${ }^{1} \cdot$ Man Updesh Singh Sachdeva ${ }^{1} \cdot$ Neelam Varma $^{1}$
}

Received: 27 January 2020 / Accepted: 20 April 2020 / Published online: 21 May 2020

(C) Springer-Verlag GmbH Germany, part of Springer Nature 2020

\begin{abstract}
With newer techniques, improvements have been made in reticulocyte counting, but variability is still there. In this study, we evaluated the three methods of reticulocyte counting, namely automated, flow cytometry, and manual methods for reticulocyte count and also for the enumeration of immature reticulocyte fraction (IRF). Reticulocyte count was done by manual method (light microscopy); two different automated analyzers, LH-780, Beckman Coulter, USA and Pentra-XLR, Horiba Ltd., Japan; and flow cytometry (FACS Canto II, B.D. Bioscience, USA). Also, the classification of stages of maturation was done, and percentage was counted based on quantity of reticulum and its distribution in the cytoplasm. Statistical analysis was done to compare statistical difference between methods. A total of 302 patient samples and 40 normal samples were included. For all automated methods, there is a tendency to overestimate with respect to microscopic methods. There is a strong correlation between manual and automated methods as well as among the 2 automated analyzers. The median IRF in LH-780 had a tendency to be on the higher side as compared with that in Pentra-XLR and manual method $(p<0.05)$. There is good correlation between the methods for reticulocyte counts. However, the normal values are dependent on the method used.
\end{abstract}

Keywords Reticulocyte $\cdot$ Automated hematology counters $\cdot$ Immature reticulocyte fraction

\section{Introduction}

The clinical laboratories currently employ two methods for reticulocyte counting: manual and automated. The traditional and standard method for reticulocyte enumeration has been manual counting by microscopy since 1940 for its advantages of simplicity and low cost. However, the analytical variables involved in it make it susceptible to an inherent imprecision and inaccuracy, because of differences in staining methods, quality of blood film, and inter-observer variations, leading to high coefficient of variation [1].

With the advent of flow cytometric analysis and fluorescent dyes in mid-1990s, reticulocyte counting found automation. Automated reticulocyte counting can be carried out by either

Electronic supplementary material The online version of this article (https://doi.org/10.1007/s12308-020-00395-8) contains supplementary material, which is available to authorized users.

Shano Naseem

shanonaseem@yahoo.co.in

1 Department of Hematology, Postgraduate Institute of Medical Education and Research, Chandigarh 160012, India an automated hematology analyzer with parameters for reticulocytes counting or a flow cytometer, using thiazole orange or acridine orange dyes. The automated methods have led to significant savings in labor costs and improvement in the accuracy and precision of the reticulocyte enumeration compared with manual counting.

The automated hematology analyzers use fluorescent or non-fluorescent dyes binding to reticulocyte RNA and subsequently enumerated based on principles like impedance, scatter, or fluorescence. In addition, automated hematology analyzers also provide various reticulocyte parameters, including immature reticulocyte fraction (IRF), reticulocyte hemoglobin content (Retic-He), and mean spherical reticulocyte volume (MSCV) to name a few $[2,3]$.

The flow cytometer for reticulocyte count uses fluorescent dyes and also allows for analysis on larger number of events, minimizing the statistical bias inherent to the low number of cells observable by microscopic methods [4].

With newer techniques, improvements have been made for low variability, but it is still there. Also, previous studies, comparing the reticulocyte count using manual method, flow cytometer, and or automated analyzers, have reported variable findings, some showing comparable results and some not [5-7]. 
In this study, we therefore, planned to evaluate the three methods of reticulocyte counting, including automated, flow cytometry, and manual methods for reticulocyte count and also for the enumeration of IRF.

\section{Material and methods}

The study complied with all the relevant national regulations and institutional policies, and is in accordance with the Helsinki Declaration, and has been approved by the institutional review board. Reticulocyte count was done by manual method (light microscopy); two different automated analyzers, LH-780, Beckman Coulter, USA, and Pentra-XLR, Horiba Ltd., Japan; and flow cytometry (FACS Canto II, B.D. Bioscience, USA).

\section{Inclusion of subjects into the study}

The study was conducted over a period of 1 year and consecutively received samples for routine hemogram, and reticulocyte counts in the department were included in the study. A total of 302 samples (from patients) and 40 samples (from normal controls) were enrolled in the study. The patient samples included the unutilized part of $3 \mathrm{ml}$ of peripheral venous blood sent for routine hemogram and reticulocyte count to the department. The normal controls were from healthy volunteers who consented for participation in the study.

In the department, routine hemogram and reticulocyte count are done on the automated analyzer, LH-780 (Beckman Coulter), and the details of reticulocyte count, hematocrit, and IRF were noted from it. Based on the reticulocyte count given by the LH-780, the cases were divided into 5 groups:

1. Group 1, with reticulocyte count, $\leq 2 \%$

2. Group 2, with reticulocyte count, $2.0-4.9 \%$

3. Group 3, with reticulocyte count, 5.0-9.9\%

4. Group 4, with reticulocyte count, 10.0-19.9\%

5. Group 5, with reticulocyte count, $\geq 20 \%$

Subsequently, the blood sample was divided into 3 parts and used for reticulocyte counting by other automated analyzer, Pentra-XLR-Horiba; manually by light microscopy; and by flow cytometry, on FACS Canto II.

\section{Exclusion of subjects from the study}

Samples which could not be processed for other 3 methods, within an hour of being run on the automated analyzer LH780 (Beckman Coulter), were excluded from the study.

\section{Methods for reticulocyte counting}

\section{Automated analyzer, LH-780, Beckman Coulter}

Following the manufacturer's instructions, the sample was run on the LH-780 analyzer.

The Beckman Coulter procedure of reticulocyte analysis uses non-fluorescent dye - new methylene blue stain - to precipitate the residual RNA within the reticulocytes. The function of the reticulocyte stain is to identify and delineate the reticulocytes from mature red cells. After aspiration, on the analyzer, the blood is mixed with the new methylene blue stain and allowed to incubate for a short period of time. An acidic, hypo-osmotic, ghosting solution is then introduced, clearing the hemoglobin while preserving the stained RNA within the reticulocytes. Once the cells have been stained and cleared, the VCS technology method of reticulocyte analysis counts and classifies reticulocytes by a flow cytometric means of cell interrogation.

\section{Automated analyzer, Pentra-XLR, Horiba}

Following the manufacturer's instructions, the sample was run on the Pentra-XLR analyzer.

The dye used for reticulocyte counting is ABX Fluocyte. It contains thiazole orange, a fluorescent stain, which is specific to nucleic acids. The binding with RNA gives an increase of fluorescence. The laser optical bench simultaneously measures the fluorescence of the cells passing through the measuring point into the flow-cell, and the volume by absorbance. The size of the cell is measured by resistivity, the scattered light (FSL) is measured approximately $200 \mathrm{uS}$ after the aperture measurement, the fluorescent signal (OFL) is measured simultaneously with the FSL.

\section{Manual count by light microscopy}

The manual platelet count was done by the method described previously, wherein equal amount of blood and $1 \%$ Azure B were mixed in $12 \times 75-\mathrm{mm}$ polystyrene tube and incubated at $37^{\circ} \mathrm{C}$ for $20 \mathrm{~min}$. Blood films were made on glass slides, and after drying, reticulocyte counting was done under a microscope using $\times 100$ objective lens, analyzing a minimum of $1000 \mathrm{RBC}$, and the count was expressed percentage [3].

Also, the classification of stages of maturation was done and percentage counted based on the quantity of reticulum and its distribution in the cytoplasm as described by Heilmeyer [3, 8].

1. Group $I=$ Dense clump reticulum is seen.

2. Group II = Reticulum forming a wreath is seen.

3. Group III = Reticulum wreath disintegrates.

4. Group IV= Only few scattered granules are seen. 


\section{Flow cytometry}

The reticulocyte analysis was done using the "Retic-Count kit" (Catalog No. 349204, BD Biosciences, USA), as per the manufacturer's instructions.

Two polystyrene tubes were used for each sample, and $0.5 \mathrm{ml}$ of BD Retic-count reagent was added to one tube (stained) and $0.5 \mathrm{ml}$ phosphate buffered saline to a second tube (unstained). A total of $2.5 \mu$ well-mixed EDTA blood was added to both tubes, mixed and incubated at room temperature for $30 \mathrm{~min}$ in dark. Subsequently, 200,000 events were acquired from both stained and unstained tubes on flow cytometer (BD Canto II). The acquisition and analysis was done using the BD Diva software.

\section{Corrected reticulocyte count (\%)}

The correction of reticulocyte count was done for the degree of anemia, so that the reticulocyte count is not spuriously elevated when it is related to the reduced number of red blood cells in an anemic patient.

The correction for the reticulocyte count by the manual method by LH-780 automated analyzer and flow cytometry was done using the following formula: corrected reticulocyte count $(\%)=\%$ reticulocytes $\times($ patient hematocrit/45) [9].

The hematocrit value given by the LH-780 automated analyzer was used for correction.

The automated analyzer ABX Pentra-XLR from Horiba itself provides "corrected reticulocyte count" to correct for variation in reticulocyte count for the degree of anemia, and this value was noted in the study for analysis for Pentra-XLR data.

\section{Statistical analysis}

Statistical analysis was carried out using Statistical Package for Social Sciences, version 22 (SPSS Inc., Chicago, USA). The data obtained through use of all assessed reticulocyte counting methods was not normally distributed. Therefore, Wilcoxon test was used to compare statistical difference between methods. Correlation was also determined using Spearman's correlation coefficient $(r)$. For method comparison, linear regression was performed. Dispersion graphs were also produced. Bias plots also known as Bland-Altman plots that plot mean difference ( $y$ axis) against the averages of two techniques were also produced wherever possible (if normality is present among the differences) using a real statistics software. $p$ values $<0.05$ were considered to be significant.

\section{Results}

A total of 302 patient samples and 40 normal samples were included. The patient samples were grouped into 5 groups on the basis of the reticulocyte count generated by the LH-780 (Beckman Coulter) automated hematology analyzer.

Most cases were in group 1-3. The overall distribution of cases according to the groups is outlined in Table 1.

Subsequently, details of reticulocyte count, namely corrected reticulocyte count (percentage), absolute reticulocyte count, and immature reticulocyte fraction (IRF), were studied and compared for the four methods used for their assessment. Comparison was also made between the associated parameters, namely, mean reticulocyte volume (MRV) and HLR\% (in LH-780), and RETH\% (in Pentra-XLR).

The data was compared in two ways: (i) Data from all subjects (302 patients and 40 normal controls) were compared with the methods for reticulocyte evaluation. (ii) Data from subgroups (groups 1 to 5) were compared with all methods.

\section{Comparison of methods studied: overall}

For all automated methods, there is a tendency to overestimate with respect to microscopic methods. There is a strong correlation between manual versus automated methods as well as among the 2 automated analyzers. Wilcoxon test indicated significant difference between methods $(p<0.05)$ except for manual versus LH-780 $(p=0.940)$. The linear regression statistics show strong correlation among the methods with a greater correlation for automated methods using fluorescent dyes than using non-fluorescent dyes. A positive intercept with a slope less than 1 agrees with tendency to overestimate at low values and to underestimate at high values for manual versus automated methods.

For absolute counts, also, same trends were observed (data not shown).

The details, are summarized in Tables 2, 3, and 4, and Fig. 1 shows the regression plots.

Figure 2 depicts the bias plots/Bland-Altman plots showing the difference versus averages of two techniques. The BlandAltman plot revealed an agreement among the assessed methods with a slight positive bias when Pentra-XLR and flow cytometry were compared with LH-780 (bias $=0.51$ and 0.46 respectively) supporting an overestimation in fluorescent methods when compared with non-fluorescent methods.

\section{Immature reticulocyte fraction}

IRF is a calculated parameter in both LH-780 and PentraXLR. In the manual method, IRF was calculated by identifying and calculating the total number of reticulocytes present in stages I to IV and subsequently using the formula.

Manual IRF $=$ stage $(\mathrm{I}+\mathrm{II}) /$ stage $(\mathrm{I}+\mathrm{II}+\mathrm{III}+\mathrm{IV})$ 
Table 1. Distribution of samples studied

Distribution of case

Patient samples $(n=302)$

\begin{tabular}{|c|c|c|c|c|c|}
\hline Groups & Reticulocyte percentage & Number of cases & Male & Female & Male:female \\
\hline Group- 1 & $\leq 1.9 \%$ & $96(31.8 \%)$ & 63 & 33 & $1.9: 1$ \\
\hline Group- 2 & $2.0-4.9 \%$ & $114(37.7 \%)$ & 54 & 60 & $1: 1.1$ \\
\hline Group- 3 & $5.0-9.9 \%$ & $65(21.5 \%)$ & 36 & 29 & $1.24: 1$ \\
\hline Group- 4 & $10.0-19.9 \%$ & $19(6.3 \%)$ & 11 & 8 & $1.3: 1$ \\
\hline Group- 5 & $\geq 20 \%$ & $8(3.6 \%)$ & 5 & 3 & $1.6: 1$ \\
\hline \multicolumn{6}{|c|}{ Normal samples $(n=40)$} \\
\hline & $0.29-2.56$ & 40 & 21 & 19 & $1.1: 1$ \\
\hline
\end{tabular}

The median in LH-780 had a tendency to be on the higher side as compared with that in the Pentra-XLR and manual method, and the difference between methods was significant $(p<0.05)$.

Linear regression showed a weak relationship with low $r$ (0.274-0.385).

The details are summarized in Supplementary Tables 1-3, and Supplementary Fig. 1 depicts the linear regression plots for IRF for the comparison of methods tested.

\section{Comparison of methods studied: within groups}

For reticulocyte count $<1.9 \%$, median reticulocyte count had tendency to be higher for automated methods when compared with that of the manual method with linear regression statistics underlying the fact that there is a tendency to overestimate the count among the automated methods when compared with manual with absolute counts following same trend.

For reticulocyte counts of 2.0-4.9\% (and the absolute counts), the trends were again similar to as in the previous group.

For reticulocyte counts of 5.0-9.9\%, the medians were comparable among manual, LH-780, and flow cytometry, but in Pentra-XLR, they tend to be a little higher. The scatter plots reveal that LH-780 underestimates when compared with the manual method.

For reticulocyte counts of $10.0-19.9 \%$, there is a reversal in the trend with the manual method median on the higher side when compared with that of the automated methods. The scatter plots reveal a tendency of automated methods to underestimate the counts when compared with the manual method and absolute numbers toeing the same line.

For reticulocyte counts $>20 \%$, the median manual count tends to be higher than automated methods with the scatter plots again revealing a tendency of automated methods to underestimate the counts when compared with the manual method.

For immature reticulocyte fraction among the groups, the median for LH-780 was higher as compared with that of the Pentra-XLR and manual methods. The linear regression analysis showed that though there is a good correlation among methods, scatter plot analysis does not reveal a good concordance between the methods.

\section{Discussion}

Reticulocyte analysis plays a pivotal role in clinical investigation for the estimation of degree of effective erythropoiesis and differential diagnosis of different anemias. With the advent of automation, which are generally based on measurement of reticulocytes after staining with either fluorescent or non-fluorescent dyes, the disadvantages associated with manual methods have been taken care of with a reduced turnout time. An additional advantage of analyzers is the associated parameters which have utility in diagnosis and differentiation of certain anemias, IRF, reticulocyte hemoglobin content, and MRV being important among them.

Table 2 Summary of statistical analysis for reticulocyte percentage(corrected) using the automated analyzers (LH-780, Pentra-XLR), flow cytometry, and manual method

\begin{tabular}{lllllll}
\hline Methods & Number of cases & Mean $(\%)$ & Std. Deviation & Data range (Min-Max) (\%) & Median (\%) & Interquartile interval \\
\hline LH-780 & 342 & 2.59 & 2.69 & $0.00-16.64$ & 1.71 & 1.92 \\
Pentra-XLR & 334 & 3.18 & 3.01 & $0.09-19.48$ & 2.20 & 2.34 \\
Flow cytometry & 342 & 3.05 & 3.12 & $0.09-18.28$ & 2.05 & 2.33 \\
Manual & 342 & 3.02 & 4.30 & $0.00-28.54$ & 1.57 & 2.29 \\
\hline
\end{tabular}


Table 3 Comparison of methods for reticulocyte percentage (corrected) using Wilcoxon test and Spearman correlation

\begin{tabular}{llllrrr}
\hline & Manual vs LH-780 & $\begin{array}{l}\text { Manual vs } \\
\text { Pentra-XLR }\end{array}$ & $\begin{array}{l}\text { Manual vs flow } \\
\text { cytometry }\end{array}$ & $\begin{array}{l}\text { LH-780 vs } \\
\text { Pentra-XLR }\end{array}$ & $\begin{array}{l}\text { LH-780 vs flow } \\
\text { cytometry }\end{array}$ & $\begin{array}{l}\text { Pentra-XLR vs } \\
\text { flow cytometry }\end{array}$ \\
\hline $\begin{array}{l}\text { Wilcoxon test } p \text { value } \\
\begin{array}{l}\text { Spearman's correlation } \\
\text { coefficient }\end{array}\end{array}$ & 0.940 & 0.000 & 0.001 & $<0.001$ & $<0.001$ & 0.731 \\
\hline
\end{tabular}

The present study compared the reticulocyte percentage (corrected), absolute reticulocyte count, and IRF for manual and automated methods.

\section{Reticulocyte percentage (corrected) and absolute reticulocyte count}

When an overall data assessment was made (patients and controls), the medians of automated reticulocyte count tend to be on the higher side as compared with the manual reticulocyte count with a tendency to overestimate, both in percentage and absolute values with respect to microscopic methods. The results obtained through Wilcoxon test revealed significant differences $(p<0.05)$ among the methods, while the Spearman's correlation coefficient estimated an overall good correlation among methods.

The regression statistics show a constant positive intercept with a slope $<1$. This agrees with tendency to overestimate at low values and to underestimate at normal and high values. Though difference between "discrete" manual methods, which measures the amount of precipitate and the automated methods, which use a "continuum" measure of RNA content can cause overestimation, this cannot completely justify the difference between the instruments. The analytic sensitivity is therefore noteworthy.

Buttarello et al. analyzed 5 different automated methods and opined that a tendency to overestimate the lower values and underestimate, in less extent, the values for samples with high reticulocyte count was present [7].

When the comparison is drawn between methods based on the dye used, values tend to be higher for XLR-Pentra analyzer and flow cytometry that employ fluorescent staining of reticulocytes for enumeration as compared with manual and
LH-780 analyzer that use azure B and new methylene blue, respectively, which are non-fluorescent dyes.

Skiemer et al. reported the methods employing fluorescent dyes produce different counts than those using nonfluorescent dyes [6]. The assessed data also confirms the results of various studies reporting the same [10-14]. The said phenomena might be caused due to strong increase of fluorescence intensity of thiazole orange in combination with RNA of reticulocytes that enhance the sensitivity for measurement, and even the reticulocytes with minimum RNA content are also detected. It is yet to be answered whether these differences are clinically relevant. Nevertheless, they should be taken in consideration as the reference values depend on the method used.

The present study also divided the obtained values from LH-780 into 5 subgroups based on the reticulocyte percentage ranging from low to high. In group $1(<1.9 \%)$ and group 2 $(2.0-4.9 \%)$, the scatter plot analysis seems to agree to the point that there is a tendency of overestimation in this group by automated methods when compared with that of manual method. In group $3(5.0-9.9 \%)$, the median was comparable among manual, LH-780, and flow cytometry, but in Pentra, they tend to be little higher and notably the LH-780 underestimates when compared with the manual method. In group 4 (10.0-19.9\%), the median reticulocyte percentage for manual was higher than automated methods, and the scatter plots confirm a tendency to underestimate at higher counts by automated methods as opposed to the manual method. In group 5 (> $20.0 \%$ ), higher median reticulocyte percentage for manual as opposed to automated methods was noted, and the scatter plots with evidence from bias plots revealed a clear underestimation of automated methods when compared with the manual method. The absolute reticulocyte counts in each group follow the same trend as the reticulocyte percentage.
Table 4 Comparison of reticulocyte percentage (corrected): linear regression statistics

\begin{tabular}{lllll}
\hline Methods & Intercept $(a)$ & Slope $(b)$ & $\begin{array}{l}\text { Correlation } \\
\text { coefficient }(r)\end{array}$ & $\begin{array}{l}\text { Determination } \\
\text { coefficient }\left(r^{2}\right)\end{array}$ \\
\hline Manual vs LH-780 & 1.013 & 0.521 & 0.831 & 0.691 \\
Manual vs Pentra-XLR & 1.227 & 0.661 & 0.917 & 0.841 \\
Manual vs flow cytometry & 1.249 & 0.597 & 0.822 & 0.676 \\
Pentra-XLR vs LH-780 & 0.044 & 0.773 & 0.907 & 0.823 \\
Flow cytometry vs LH-780 & 0.478 & 0.691 & 0.800 & 0.640 \\
Pentra-XLR vs flow cytometry & 0.396 & 0.976 & 0.912 & 0.831 \\
\hline
\end{tabular}



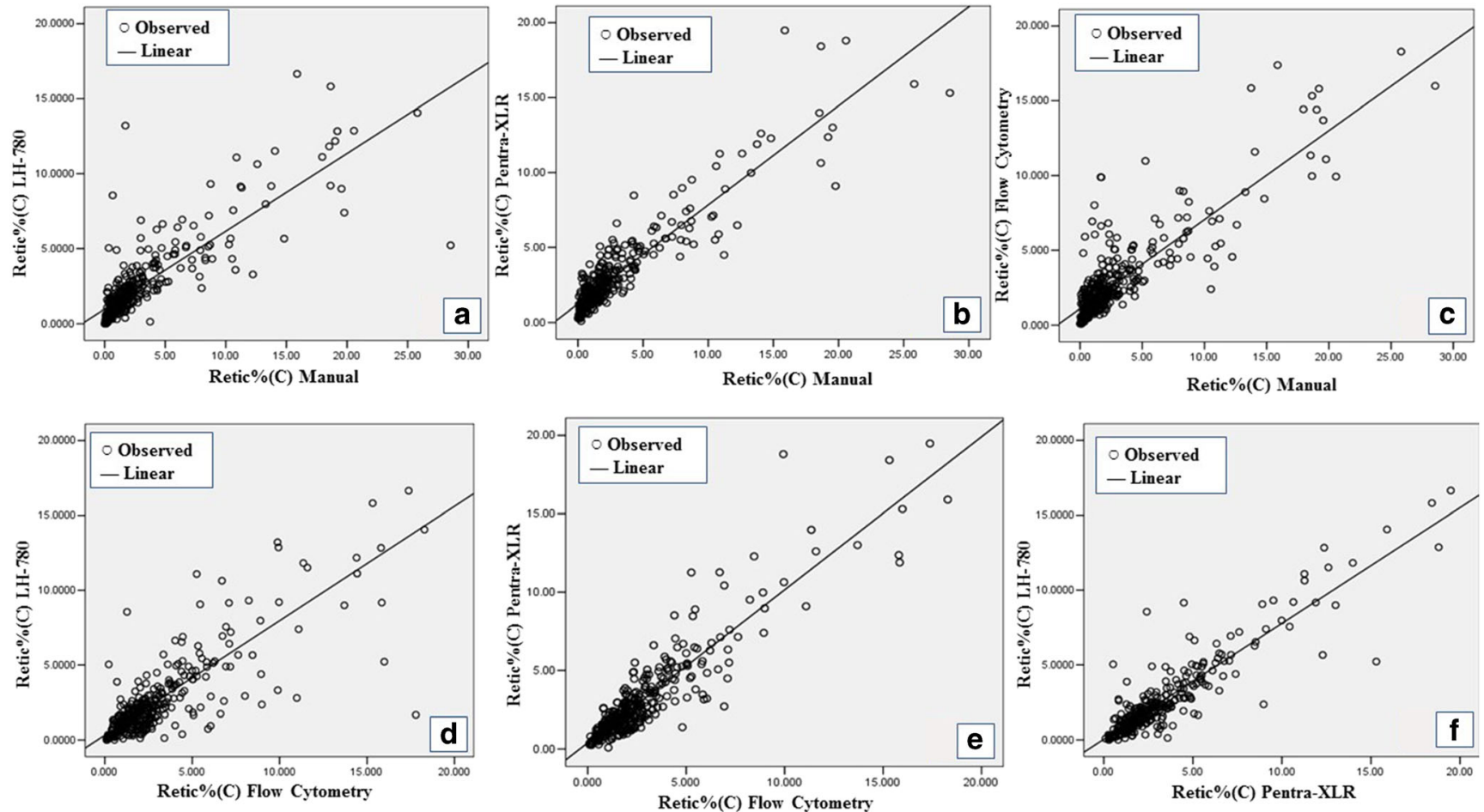

Fig. 1 Regression plot showing corrected reticulocyte counts between different methods. a LH-780 versus manual. b Pentra-XLR versus manual. $\mathbf{c}$ Flow cytometry versus manual, d LH-780 versus flow cytometry. e Pentra-XLR versus flow cytometry. f LH-780 versus Pentra-XLR
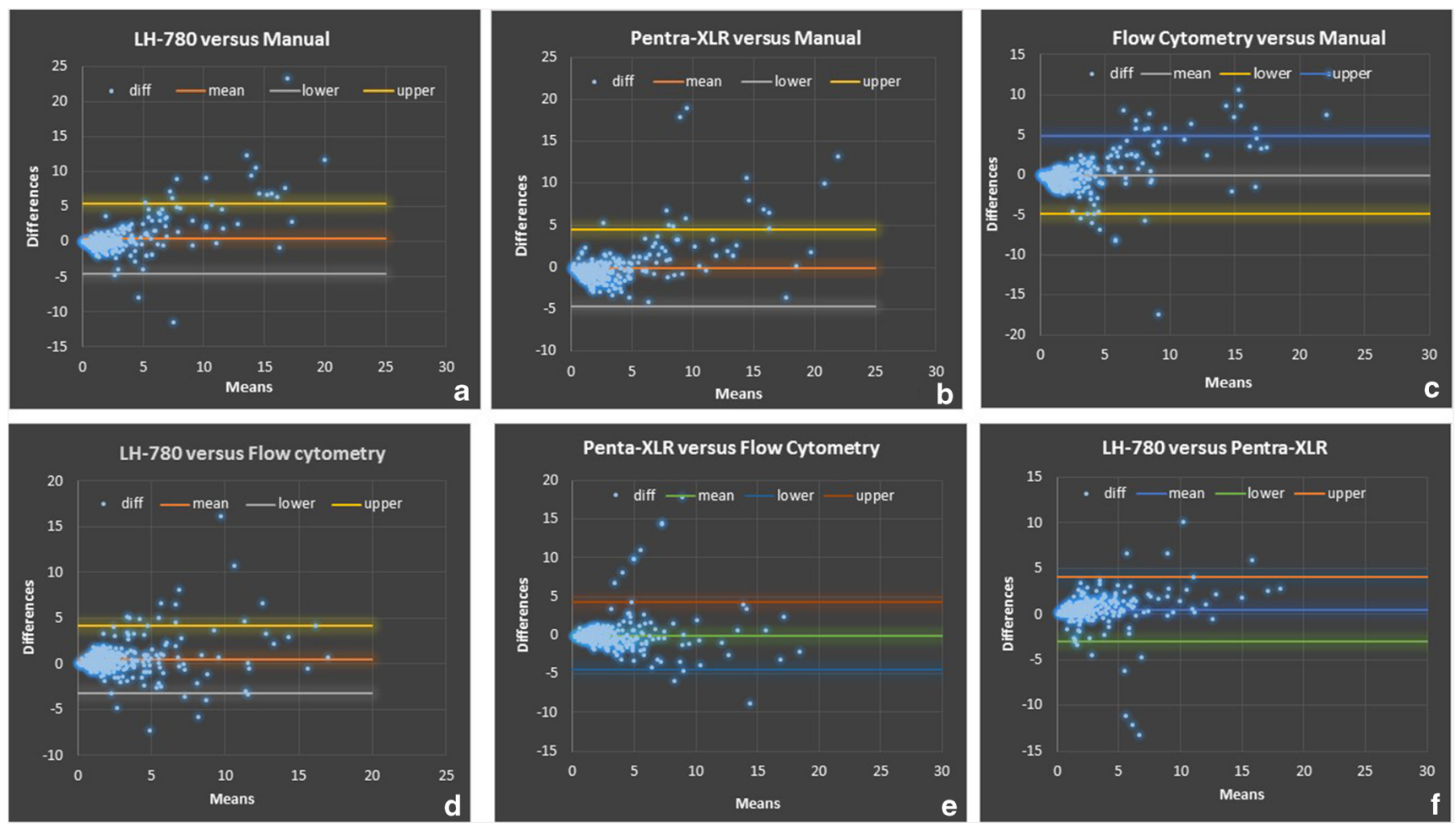

Fig. 2 Bland-Altman plots showing the difference versus means of two techniques. a LH-780 versus manual. b Pentra-XLR versus manual, $\mathbf{c}$ Flow cytometry versus manual, d LH-780 versus flow cytometry. e Pentra-XLR versus flow cytometry. f LH-780 versus Pentra-XLR 


\section{Immature reticulocyte fraction}

The term refers to least mature or the younger fraction of reticulocytes which assesses the erythropoietic activity. A particularly useful application of IRF in cases of reticulocytopenia is the identification of early marrow regeneration following bone marrow transplantation or chemotherapy noted by the reappearance of reticulocytes with a high RNA content and, therefore, by an increase in the IRF [15]. A general principle used in automation for enumerating IRF is to divide the reticulocytes into subpopulations (2 or 3) according to the RNA content and, therefore, the different maturity levels. These are detected by proprietary techniques.

The methods in our study, namely LH-780, divide reticulocyte into two fractions (or 10 regions) and Pentra-XLR into 3 fractions. For manual method, Heilmeyer classification was used and reticulocytes segregated into four groups with group I comprising most immature reticulocyte.

Comparison for IRF obtained from LH-780, ABX Pentra, and manual method was performed. An overall data comparison revealed higher IRF values for LH-780 as compared with that of the manual method and Pentra-XLR with significant differences among the methods. A weak correlation is observed between manual versus LH-780 and LH-780 versus Pentra, while a moderate correlation is observed between manual versus Pentra. Within group comparison also reveals the same trend with only variation being in group with $>20 \%$ reticulocytes where the difference between the methods is not significant.

The regression analysis also gives coefficient of correlation in range from 0.27 to 0.38 in overall data assessment while within groups from 0 to 0.34 , exception being the group with $>20 \%$ reticulocytes where it ranged from 0.518 to 0.718 . The scatter plot analysis clearly provides a strong evidence to low $r$ values identifying a very low concordance among the methods. It is very obvious that the entities measured by the assessed methods for calculating IRF are not same.

Lacombe et al. also opined that it was impossible for IRF to have a good concordance among instruments where they compared Sysmex R-2000 and ABX Pentra 120 Retic and got similar low $r$ values [16]. Buttarello et al. performed a similar analysis on 5 different instruments using methods based on fluorescent and nonfluorescent methods. They concluded that IRF values obtained do not offer the same information content although its usefulness was confirmed by all methods [7].

The lack of a "universal" stable reference material and of a reference method for quantifying the IRF with definition of IRF being different for different methods makes it difficult in standardizing the parameter.

\section{Conclusion}

In the present study, comparative evaluation of 4 methodsmanual method; flow cytometry; and automated analyzers, Beckman Coulter, LH-780, and Pentra-XLR — was done, for assessing reticulocyte counts and the associated parameters on 342 samples.

The results showed:

1. A good correlation among all four methods.

2. However, the results differed with the automation and dye used for enumeration.

3. There is a tendency to overestimate at low reticulocyte counts and underestimate at higher reticulocyte counts by the automated methods when compared with the manual method.

4. Fluorescent dye labeling increases the sensitivity thus resulting in higher values as compared with nonfluorescent dyes.

5. Flow cytometry and ABX Pentra-the methods give concordant results as they are using same fluorescent dye.

6. Manual method and LH-780 - the methods give concordant results as they both use a non-fluorescent dye.

The overall data indicates that there is good correlation between the methods for reticulocyte counts. However, the normal values are dependent on the method used.

The associated parameter-IRF, MRV, and high-scatter reticulocyte \% - cannot be used interchangeably, and there is also a lack of universal reference material for these parameters. The reference intervals for these parameters should be method-specific, especially for IRF, since it has a great clinical utility.

Acknowledgments The authors sincerely acknowledge the support rendered by Horiba India Pvt. Ltd., by providing the automated analyzer, Pentra-XLR, and the reagents for this study.

\section{Compliance with ethical standards}

Conflict of interest The authors declare that they have no conflicts of interest.

\section{References}

1. Riley RS, Ben-Ezra JM, Goel R, Tidwell A (2001) Reticulocytes and reticulocyte enumeration. J Clin Lab Analysis 15:267-294

2. Smock KJ, Perkins SL. Examination of blood and bone marrow. In: Greer JP, Arber DA, Glader B, et al (eds). Wintrobe's clinical hematology 2011; 13th edition. Lippincott Williams and Wilkins, Philadelphia, pg 1-18

3. Briggs C, Bain BJ. Basic haematological techniques. In. Bain BJ, Bates I, Laffan MA, et al (eds). Dacie and Lewis practical hematology 2011; 11th edition. Elsevier Churchchill Livingstone, London, pg. $24-56$ 
4. Corberand JX (1996) Reticulocyte analysis using flow cytometry. Hematol Cell therapy 38:487-494

5. Van Petegem M, Cartuyvels R, de Schouwer P, van Duppen V, Goossens W, van Hove L (1993) Comparative evaluation of three flow cytometers for reticulocyte enumeration. Clin Lab Haematol 15:103-111

6. Siekmeier R, Bierlich A, Jaroß W (2000) Determination of reticulocytes: three methods compared. Clin Chemistry Lab Medicine 38: 245-249

7. Buttarello M, Bulian P, Farina G, Temporin V, Toffolo L, Trabuio E, Rizzotti P (2001) Flow cytometric reticulocyte counting. Am J Clin Pathol 115:100-111

8. Heilmeyer L. (1931) Blutfarbostoffwechselstudien. Probleme, Methoden, und Kritik der Whippleschen Theorie. Dtsch Arch Klein Med. 171

9. Means Jr RT, Glader B. Anemia: general considerations. In. Greer JP, Arber DA, Glader B, List AF, Means Jr RT, Paraskevas F, et al (eds). Wintrobe's Clinical Hematology 2014; 13th edition, Lippincott Williams \& Wilkins, Philadelphia, pg. 587-616

10. Davis BH, Bigelow NC, Koepke JA, Borowitz MJ, Houwen B, Jacobberger JW, Pierre RV, Corash L, Ault KA, Batjer JD (1994) Flow cytometric reticulocyte analysis. Multi institutional interlaboratory correlation study. Am J Clin Pathol 102(4):468-477
11. Aulesa C (1993) Evaluation of the Sysmex R-3000 automated reticulocyte analyzer. Sysmex J Int 3:105-115

12. Schimenti KJ, Lacerna $\mathrm{K}$, Wamble A, Maston L, Iaffaldano C, Straight M, Rabinovitch A, Lazarus HM, Jacobberger JW (1992) Reticulocyte quantification by flow cytometry, image analysis, and manual counting. Cytometry. 13(8):853-862

13. Profile I (1994) Retikulozytenzählung im Blut mit demCoulter® STKS und dem Coulter ${ }^{\circledR}$ ERICS® Profile II. Lab Med 18(323): 323

14. Rudensky B (1997) Comparison of a semi-automated new Coulter methylene blue method with fluorescence flow cytometry in reticulocyte counting. Scand J Clin Lab Invest 57(4):291-296

15. Piva E, Brugnara C, Spolaore F, Plebani M (2015) Clinical utility of reticulocyte parameters. Clin Lab Med 35(1):133-163

16. Lacombe F, Lacoste L, Vial J-P, Briais A, Josy R, Boisseau MR, Bernard P (1999) Automated reticulocyte counting and immature reticulocyte fraction measurement: comparison of ABX PENTRA 120 Retic, Sysmex R-2000, flow cytometry, and manual counts. Am J Clin Pathol 112(5):677-686

Publisher's note Springer Nature remains neutral with regard to jurisdictional claims in published maps and institutional affiliations. 